\title{
Cross-activity of honeybee queen mandibular pheromone in bumblebees provides evidence for sensory exploitation
}

\author{
Sarah A. Princen ${ }^{1}$, Annette Van Oystaeyen ${ }^{1,2}$, Clément Petit ${ }^{2,3}$, Jelle S. van Zweden ${ }^{1}$, Tom \\ Wenseleers $^{1^{*}}$ \\ ${ }^{1}$ Department of Biology, KU Leuven, Laboratory of Socioecology and Social Evolution, Leuven, Belgium \\ 2 Biobest Group NV, 2260 Westerlo, Belgium \\ ${ }^{3}$ Montpellier SupAgro, 34060 Montpellier. France \\ * Corresponding author: Address: Naamsestraat 59, 3000 Leuven, Belgium. Tel: +3216 323964, E-mail: \\ tom.wenseleers@kuleuven.be
}

Short title: 'Honeybee pheromones inhibit bumblebee reproduction'

\section{ABSTRACT}

The evolutionary origin of queen pheromones, which regulate reproductive division of labor in insect societies, has been explained by two evolutionary scenarios: the sender-precursor hypothesis and the sensory exploitation hypothesis. These scenarios differ in terms of whether the signaling system was built on preadaptations on the part of either the sender queens or the receiver workers. While some social insect queen pheromones - such as cuticular hydrocarbons - were likely derived from ancestral fertility cues and evolved according to the former theory, the honeybee's queen mandibular pheromone (QMP) has been suggested to act directly on pre-existing gene-regulatory networks linked with reproduction. This is evidenced by the fact that QMP has been shown to also inhibit ovary activation in fruit flies, thereby implying exploitation of conserved physiological pathways. To verify whether QMP has similar effects on more closely related eusocial species, we here tested for QMP crossactivity in the bumblebee Bombus terrestris. Interestingly, we found that the non-native QMP blend significantly inhibited egg-laying in both worker and queen bumblebees and caused accompanying shifts in ovary activation. The native bumblebee queen pheromone pentacosane, by contrast, only inhibited the reproduction of the workers. Overall, these findings support the hypothesis that honeybee QMP likely evolved via a route of sensory exploitation. We argue that such exploitation could allow social insect queens to produce compounds that manipulate the workers to remain sterile, but that a major hurdle would be that the queens themselves would have to be immune to such compounds. 
Keywords: social insects, queen pheromones, sensory exploitation, queen control, honeybees, Bombus terrestris 


\section{INTRODUCTION}

A major paradox in the evolution of animal signals is to explain how two traits, namely producing the signal on the part of the sender and effectuating an adaptive response on the part of the receiver, could arise in the population seemingly simultaneously (Bradbury and Vehrencamp, 2011; Stökl and Steiger, 2017; Wyatt, 2014). A resolution to this paradox is provided by two different scenarios which posit that signals always evolve from pre-existing traits - so-called "preadaptations" - on the part of either the senders or the receivers (Bradbury and Vehrencamp, 2011; Stökl and Steiger, 2017; Wyatt, 2014). In particular, one scenario - the sender-precursor hypothesis - proposes that signals are derived from cues that were already being produced as functionless physiological by-products or to serve another unrelated purpose (Fig. 1; Stökl and Steiger, 2017; Wyatt, 2014). These cues would then act as preadaptations on the part of the senders and allow for the evolution of a full signal if the receivers evolved a way to detect and optimally respond to the provided cues. By contrast, the sensory exploitation hypothesis posits that animal signals could also evolve by building on preadaptations on the part of the receivers, for example by exploiting parts of their physiology

(Fig. 1; Peso et al., 2014; Stökl and Steiger, 2017; Wyatt, 2014). In the present study, we investigate which of these two scenarios is most likely in the context of the evolution of social insect queen pheromones, which regulate these insects' reproductive division of labor (Holman, 2018; Le Conte and Hefetz, 2008; Oi et al., 2015; Van Oystaeyen et al., 2014).

Queen pheromones are emitted by the queens and keep the workers from reproducing in her presence (Holman, 2018; Le Conte and Hefetz, 2008; Oi et al., 2015; Van Oystaeyen et al., 2014).

In the context of the evolution of social insect queen pheromones (QPs), the senderprecursor hypothesis has been most widely discussed and appears to be supported for cuticular hydrocarbon QPs, which have recently been identified as a conserved class of queen pheromones across several independently evolved lineages of social insects, including ants, wasps, honeybees and bumblebees (Holman, 2018; Princen et al., 2019; Van Oystaeyen et al., 2014). Some ants and wasps, for example, were found to employ identical or structurally related long-chain linear and methyl-branched alkanes as queen pheromones that partly inhibit the workers' reproduction (Holman, 2018; Van Oystaeyen et al., 2014). Likewise, in bumblebees, treatment with the queen-characteristic hydrocarbon pentacosane caused a decrease in worker oocyte number and an increase in oocyte resorption in Bombus terrestris (Holman, 2014; Van Oystaeyen et al., 2014), and tended to decrease and delay egg-laying in 
B. impatiens (Holman et al., 2017). The most likely explanation for this high degree of conservation is that the hydrocarbon QPs across these different groups were derived from ancestral fertility-linked cues that were already being produced by egg-laying females in common solitary ancestors (Chapuisat, 2014; Oi et al., 2015; Van Oystaeyen et al., 2014). This hypothesis is supported by the fact that in several solitary arthropods the cuticular hydrocarbon patterns of egg-laying females are clearly distinct from those of non-egg-laying ones (reviewed in Blomquist, 2010; Blomquist and Bagnères, 2010; Oi et al., 2015) as well as by phylogenetic reconstructions showing that some of the queen signals of corbiculate bees are likely derived from fertility cues that were already present in their direct solitary ancestors (Oliveira et al., 2015). In addition, a recent experimental study reported that fertility-linked hydrocarbon cues were clearly present in a primitively eusocial polistine wasp even though they were not active as queen pheromones, thereby suggesting that queen pheromones in highly eusocial insects may initially have been produced as fertility-linked cues that lacked any signaling function (Oi et al., 2019).

Under the sender-precursor hypothesis, QPs would intrinsically be honest signals of the queen's true fecundity and the workers would be expected to respond to such signals to the extent that they could gain indirect fitness from doing so (Keller and Nonacs, 1993; Kocher and Grozinger, 2011; Moritz and Crewe, 2018; Oi et al., 2015; Peso et al., 2014). The argument here is that workers could be selected to respect a queen signal and refrain from reproduction when the inclusive fitness benefits they would gain from it, e.g. by helping the queen to produce more sisters (Hammond and Keller, 2004), would outweigh the direct fitness cost of refraining from reproduction (Oi et al., 2015; Wenseleers et al., 2004a; Wenseleers et al., 2013; Wenseleers et al., 2004b; Wenseleers and Ratnieks, 2006a). The fact that worker reproduction in many species, such as bumblebees and honeybees, is punished by targeted aggression or made unprofitable by selective egg cannibalism ('policing') also contributes to the alignment of the queen and worker interests (Ratnieks and Wenseleers, 2008; Wenseleers et al., 2004a; Wenseleers and Ratnieks, 2006b).

As a corollary to the sender-precursor hypothesis, the sensory exploitation hypothesis proposes that pheromone signals could also have evolved by building on preadaptations on the part of the receivers (Fig. 1; Peso et al., 2014; Stökl and Steiger, 2017; Wyatt, 2014). More specifically, in the context of QPs, it has been proposed that the queen-worker signaling system could have come about through the exploitation of pre-existing gene-regulatory networks (GRNs) that are linked with the regulation of reproduction in the receiver workers 
(Beggs et al., 2007; Beggs and Mercer, 2009; Camiletti et al., 2013; Croft et al., 2017; Kocher and Grozinger, 2011; Oi et al., 2015). In this case, a queen pheromone could still be honest, but the sender could also potentially exploit the existing GRNs to deceive or manipulate the receiver workers (Keller, 2009; Kocher and Grozinger, 2011; Stökl and Steiger, 2017). In fact, under this scenario, the queen would be capable - at least transiently - of manipulating the workers' reproduction. In the long run, this could then either lead to a queen-worker arms race and to workers evolving counter-adaptations to escape queen control (Keller, 2009; Keller and Nonacs, 1993; Kocher and Grozinger, 2011; Oi et al., 2015; Peso et al., 2014), or to the stable maintenance of this signaling system if worker sterility provided large enough indirect fitness benefits at a limited personal cost (Oi et al., 2015; Ratnieks and Wenseleers, 2008; Wenseleers et al., 2004a; Wenseleers et al., 2013; Wenseleers et al., 2004b). Recent evidence suggests that honeybee queen mandibular pheromone (QMP) - a honeybee-specific volatile pheromone blend consisting mainly of (2E)-9-oxo-dec-2-enoic acid (9-ODA), two enantiomers of (2E)-9-hydroxydec-2-enoic acid (9-HDA), methyl 4-hydroxybenzoate (HOB) and 4-hydroxy-3-methoxyphenylethanol (homovanillyl alcohol, HVA) (Crewe and Velthuis, 1980; Plettner et al., 1997; Slessor et al., 1988) - is a likely example of a pheromone that exploits pre-existing physiological pathways. Honeybee QMP serves diverse roles, acting among others - as a sex attractant and a sterility-inducing queen pheromone (Hoover et al., 2003; Oi et al., 2015; Princen et al., 2019; Slessor et al., 2005).

A first line of evidence suggesting this possible origin for QMP is that its compound HVA was found to directly act on dopamine receptors to suppress the workers' reproduction, thereby entirely bypassing their sensory system (Beggs et al., 2007; Beggs and Mercer, 2009). A second line of evidence was provided by the unexpected discovery that honeybee QMP also inhibits reproduction in the phylogenetically distantly related fruit fly Drosophila melanogaster, in which it reduces ovary size, the number of eggs that were laid and the number of viable offspring when administered to virgin females (Camiletti et al., 2016; Camiletti et al., 2013; Galang et al., 2019). The QMP hydroxy-acids were the main compounds eliciting this anti-ovarian response in fruit flies, even though a hydroxy-acid that occurs in workers, 10-HDA, also inhibited reproduction (Galang et al., 2019). In addition, QMP was found to attract male fruit flies and enhance their sexual response (Croft et al., 2017). These results suggest that the honeybee QMP blend may have evolved by exploiting pre-existing, highly conserved physiological pathways linked with the regulation of ovary development and sexual signaling (Camiletti et al., 2013; Croft et al., 2017). 
To further test the theory that honeybee QMP could have evolved via a route of sensory exploitation, we here verify whether honeybee QMP also inhibits reproduction in a eusocial insect, the buff-tailed bumblebee Bombus terrestris, where QMP does not natively occur (Kocher and Grozinger, 2011). Although this experiment would perhaps not be able to conclusively decide whether or not QMP evolved via a route of sensory exploitation (in fact, as we mentioned above, there is also some evidence for the sender-precursor hypothesis), it does at least allow us to test whether the effect of QMP on the reproduction of $D$. melanogaster fruit flies (Camiletti et al., 2016; Camiletti et al., 2013; Galang et al., 2019) also extends to more closely related eusocial species. Furthermore, it allows us to exclude the possibility that the observed response in fruit flies could have been merely coincidental. We expected that if honeybee QMP evolved via a route that exploited conserved reproductionrelated physiological pathways, it should show clear cross-activity and inhibit egg-laying and ovary activation also in worker and possibly even queen bumblebees. We then discuss our results within the context of competing theories over the mode of action of social insect queen pheromones, and the question of whether queen pheromones could ever be based on a manipulative mode of action, which appears plausible under a scenario of sensory exploitation (Keller, 2009; Keller and Nonacs, 1993; Kocher and Grozinger, 2011; Oi et al., 2015; Peso et al., 2014; Villalta et al., 2018).

\section{MATERIAL AND METHODS}

\section{Bumblebee rearing}

To test the effects of QMP and pentacosane on worker reproduction, ten queenright Bombus terrestris colonies (each containing at least 60 workers) were obtained from Biobest Group NV (Westerlo, Belgium) and split into three queenless subcolonies each to be exposed to one of three treatments (QMP, pentacosane and solvent control). All colonies were pre-competition point, i.e. the workers had not started to overtly compete over male production. The queenless worker subcolonies were kept in wooden nest boxes $(18 \mathrm{~cm} \times 16.5 \mathrm{~cm} \times 12 \mathrm{~cm})$ with a plexiglass lid provided with ventilation holes. Each subcolony was initially made to contain around 20 (mean: 19.85 SE: 0.12) workers and an equal amount of nest material and brood of every stage, originating from the source colony. We used workers with a natural age distribution and let new workers emerge during the experiment, as this would result in the most realistic levels of behavioural variation. To test the effect on the reproduction of queens we used ten founding queens per treatment, which were kept along with young brood (eggs 
and larval brood) in plastic nest boxes $(15.5 \mathrm{~cm} \times 15.5 \mathrm{~cm} \times 10 \mathrm{~cm})$. The nest boxes were kept in a climate room at $25^{\circ} \mathrm{C}(16 \mathrm{~L}: 8 \mathrm{D})$. All colonies were fed ad libitum with honeybee-collected pollen, which was sterilized by gamma irradiation and tested negative on pesticide residue, and had constant access to $50^{\circ}$ Biogluc $^{\circledR}$ via a wick system (all bees, $50^{\circ}$ Biogluc $^{\circledR}$ and pollen were provided by Biobest Group NV, Westerlo, Belgium).

\section{Bioassays}

Each worker subcolony or founding queen was randomly assigned to either the QMP treatment, the pentacosane treatment or an acetone solvent control. We used synthetic pentacosane and a synthetic QMP blend consisting of the four major compounds, of which one queen equivalent (the amount typically present on a single queen) comprised $250 \mu \mathrm{g} 9$ ODA, $150 \mu \mathrm{g}$ 9-HDA (82.5\% - and 17.5\% + isomers), $20 \mu \mathrm{g} \mathrm{HOB}$ and $2 \mu \mathrm{g}$ HVA (data provided by the supplier, Contech). Each day, we administered a $200 \mu \mathrm{l}$ (worker subcolonies) or $50 \mu \mathrm{l}$ (queens) acetone solution, each containing the same total dose of $200 \mu \mathrm{g}$ of either QMP or pentacosane, or only the solvent for the control. This also corresponds with roughly similar amounts when expressed in terms of queen equivalents (ca. 0.47 to 0.66 for QMP in $A$. mellifera, based on quantifications are provided to us by Contech and Hoover et al., 2003, and 0.86 for pentacosane in B. terrestris, Van Oystaeyen et al., 2014) and a dosage that is well within the range used in previous experiments (cf. Table S2 in Holman et al., 2017). The treatments were administered daily for seven or ten consecutive days in the worker subcolonies and queens respectively, by pipetting the treatment solution on top of the pupae in the nests, where natural contact with queen pheromones was expected to be the highest. No adverse effects of the acetone solvent were observed, and the overall worker mortality rates were also very low, as we observed an average cumulative total of just 2.20 dead workers per nest over our seven day experiment period (95\% CL $[1.54,3.16])$ in nests that at the end of the experiment contained an average of ca. 30 workers.

\section{Behavioral observations}

Given that reproduction by bumblebee workers is also heavily regulated and influenced by aggressive behavioral interactions between the workers (Amsalem et al., 2015), we made daily behavioral observations of all nests during the full seven-day experimental period, to be able to test the possibility that either the native queen pheromone pentacosane (Holman, 2014; Holman et al., 2017; Van Oystaeyen et al., 2014) or the non-native honeybee queen 
pheromone QMP would influence the formation of reproductive hierarchies among the bumblebee workers. Individual behavioral observation sessions lasted eight minutes per subcolony per day and were all carried out by a single observer, blind with regard to treatment and in random order. Aggressive interactions (chasing/butting, pushing/bumping, biting/grasping, buzzing, egg policing) (Sibbald and Plowright, 2014; Sibbald and Plowright, 2015) and egg-laying were counted as they were performed and summed. For behaviors that had long durations, the maximum number of workers that were seen performing these tasks was recorded and counted once for any given observation period. The latter was the case for nursing, defined as incubating brood, feeding larvae or inspecting brood cells, and foraging, which happened within the nest box and was defined as flying, crawling towards the ceiling of the box or foraging for pollen or $50^{\circ} \mathrm{Biog}_{\mathrm{uc}} \mathrm{C}^{\circledR}$, which were provided on one side of the box. Workers not performing any of these behaviors were recorded but were not included as a behavioral class in the data analysis.

\section{Dissections}

After seven and ten days, respectively, the workers and founding queens were frozen before dissection and all eggs they had laid were counted (queens were given three days longer to reproduce to make sure that the total number of eggs laid in the control condition was comparable with that seen in the worker groups and the overall statistical power to detect an effect on reproduction comparable across both experiments). Young, newly emerged workers in the worker subcolonies (lighter coloration) were discarded as they would all have been prereproductive. Ovary activation was assessed via dissection, blind with respect to treatment, and for workers was measured using the scale of Duchateau and Velthuis (1989). Ovaries with development stages of II or higher (i.e. with clearly discernible, growing oocytes) were considered as activated. We also measured head width and the mean length of the front wings' marginal cells of each dissected worker, which were then conflated into a single body size measure calculated from the first principal component of a PCA (Fig. S1a). For queens, the level of oocyte development was measured based on the number of viable mature oocytes in their ovaries.

\section{Statistical analyses}

Worker ovary activation (proportions of workers with activated ovaries) and aggressive behavior (the frequency of aggressive interactions in proportion to all scored behavior) (Tables 
S2a and S3a) were compared between treatments using binomial generalized linear mixed models (GLMM). The number of eggs laid by workers and queens (Tables S1a and S4a) and the number of viable oocytes in queens (Table S5a) were tested using Poisson GLMMs (R package Ime4; Bates et al., 2014). Colony and nest (subcolony) were added as nested random intercepts in all worker models, as was queen $I D$ in the founding queen models. Nest and queen $I D$ in these models represent so-called observation-level random effects and allowed us to account for possible overdispersion. Additionally, total number of workers (total number of workers present in the nest at the end of the experiment $=\mathrm{nr}$. of workers present initially $+\mathrm{nr}$. of newly emerged workers $-\mathrm{nr}$. of workers that died) and worker mortality (number of dead individuals observed per day per nest) were included as covariates for all worker models. Random slope models were also fitted, whereby treatment effects were allowed to vary randomly over colonies, but in all cases these reduced the fit of the models based on their AIC value, so these analyses are only given in our supplemental R script. Lastly, to exclude the possibility that the effect of QMP on the reproduction of the workers could have been caused by a toxicity effect, we compared the number of dead workers at the end of the seven-day period between treatments using a Poisson GLMM in which colony and nest were coded as nested random intercepts and total $n r$. of workers (live plus dead ones) was included as an additional covariate ( $c f$. supplemental R-script). Since our a priori hypotheses were clearly directional, and only a decrease in ovary development, egg-laying and aggressive behavior in response to the queen pheromone treatments were expected (Holman, 2018), we used onesided tests (and one-sided 95\% confidence intervals, i.e. 95\% confidence bounds) throughout, followed by control-treatment post hoc comparisons with FDR correction for multiple testing. The use of one-tailed tests is advised for directional hypotheses and avoids an unnecessary increase in the false negative rate (Cho and Abe, 2013; Murphy, 2018). In addition, our usage of one-tailed tests and one-sided $p$ values matches the stringent criteria for their use given by Ruxton \& Neuhäuser (2010): (1) we would like to carry out a directional hypothesis test as only an inhibition of worker reproduction would support the sensory exploitation hypothesis. (2) An effect in the opposite direction would falsify the sensory exploitation hypothesis to the same extent as an effect in the expected direction that was not strong enough to reject the null hypothesis. A last benefit of using one-tailed directional test is that the obtained onesided $p$-values can readily be converted to Bayes factors $K$ for the support of an effect in the expected direction versus the support for no effect or an effect in the opposite direction under 
a symmetric (uninformative) prior, since $K=(1-p) / p$ where $p$ is the one-tailed $p$ value (Jeffreys, 1961; Kass and Raftery, 1995; Marsman and Wagenmakers, 2017).

\section{RESULTS}

As expected by the hypothesis that honeybee QMP evolved by the sensory exploitation route, QMP showed clear cross-activity in the bumblebee and significantly reduced egg-laying of both workers (ratio $=1.86,95 \% C B[1.32,3.15], p=0.003$, Fig. $2 A \&$ Tables S1a,b) and queens (ratio $=1.64,95 \%$ CB $[1.16,2.81], p=0.019$, Fig. 3a \& Tables S4a,b) and significantly inhibited both worker ovary activation (odds ratio $=1.58,95 \% C B[1.10,2.86], p=0.043$, Fig. 2B \& Tables S2a,b) and queen oocyte development (ratio $=2.78,95 \% C B[1.53,14.74], p=0.014$, Fig. 3B \& Tables $5 a, b)$. The overall combined significance of QMP inhibiting egg-laying across both workers and queens was $p=0.0006$ (Fisher's method, $\chi^{2}=19.46, d f=4, c f$. supplemental $\mathrm{R}$ script). It is unlikely that the observed QMP effect is based on coincidental toxicity, since the average worker mortality (cumulative number of dead workers observed over the seven day period of our experiment) was not affected by the QMP treatment (ratio $=0.98,95 \%$ CB [0.65, 2.38], $p=0.48$, Tables $\mathrm{S} 6 \mathrm{a}, \mathrm{b})$. At the same time, sublethal toxic effects cannot be ruled out, and indeed such an effect could well represent one proximate way in which reproduction was affected in the context of an evolutionary scenario of sensory exploitation.

Observed effects for the native queen pheromone pentacosane were different from the ones seen for QMP. As expected, pentacosane significantly reduced worker egg-laying (ratio $=1.45,95 \% C B[1.03,2.47], p=0.038$, Fig. 2A \& Tables S1a,b), and this was accompanied by a nonsignificant trend to have reduced rates of worker ovary activation (odds ratio $=1.42,95 \%$ $C B[0.98,2.54], p=0.061$, Fig. 2B \& Tables S2a,b). In contrast to QMP, however, pentacosane did not significantly affect either queen egg-laying (Fig. 3A \& Tables S4a,b) or queen oocyte development (Fig. 3B \& Tables S5a,b). We should note that although the effect of pentacosane on worker ovary activation was modest in terms of significance level, the effect size (odds 1.42 fold reduced for workers to have activated ovaries when exposed to pentacosane) is contained within the $95 \%$ confidence intervals of two earlier studies (Van Oystaeyen et al., 2014: odds ratio 95\% CL [1.32, 4.09]; Holman, 2014: odds ratio 95\% $C L[1.18,4.13]$ and highly significant across all three studies combined (Fisher's method, $\chi^{2}=19.46, d f=6, p>0.001, c f$. supplemental R script). In addition, expressed as a Bayes factor, a one-tailed $p$ value of 0.061 still corresponds with a Bayes factor $K$ of $(1-0.061) / 0.061=15$, implying that based on our 
data, there is 15 times more support for pentacosane inhibiting worker ovary activation than for it having either no effect or enhancing worker ovary activation under a symmetric prior (Jeffreys, 1961; Kass and Raftery, 1995; Marsman and Wagenmakers, 2017). In Bayesian statistics, such an effect (Bayes factor $K>10$ ) is generally regarded as a strong effect (Jeffreys, 1961; Kass and Raftery, 1995).

Regarding the effects of covariates included in the aforementioned models (cf. Tables S1-S3), the standardized total number of workers and worker mortality did not significantly affect egg-laying in workers $(\beta=-0.03,95 \% C B[-0.17,0.11]$ and $\beta=-0.04,95 \% C B[-0.18,0.10]$, Table S1a) but did significantly reduce the proportion of workers with activated ovaries $(\beta=-0.61,95 \% C B[-0.81,-0.41]$ and $\beta=-0.29,95 \% C B[-0.47,-0.11], p<0.001$ and $p=0.005$ resp.) and furthermore, as might be expected, larger workers were significantly more likely to have activated ovaries (Table S2a, for discussion see Figs. S1-S3). Finally, in terms of the behavioral response to the queen pheromones, we observe that only the native queen pheromone pentacosane (odds ratio $=1.57,95 \% C B[1.14,2.54], p=0.019$ ) but not the nonnative pheromone QMP (odds ratio $=0.95,95 \% C B[0.70,1.49], p=0.60$ ) induced a significant drop in aggressive behavior linked with worker-worker competition over reproduction (Fig. 2C; Tables S3a,b). Aggressive behavior also showed a decrease in function of colony size (standardized total number of workers) ( $\beta=-0.18,95 \% C B[-0.34,-0.02], p=0.03$, Table S3a, Fig. S4).

\section{DISCUSSION}

Our results show that honeybee QMP inhibits the reproduction and ovary activation of $B$. terrestris workers and queens, with observed levels of inhibition being only slightly less than those observed in honeybee workers (Hoover et al., 2003; Princen et al., 2019) and similar to those induced in virgin D. melanogaster females (Camiletti et al., 2014; Camiletti et al., 2016; Camiletti et al., 2013; Galang et al., 2019). We further showed that the observed inhibitory effect of QMP is probably not based on coincidental toxicity, since there were no significant effects on worker mortality, even though sublethal effects would remain possible. Overall, these observations are fully consistent with the hypothesis that QMP initially evolved via a route of sensory exploitation (Fig. 1), i.e. via the exploitation of pre-existing, highly conserved physiological pathways associated with reproduction (Beggs et al., 2007; Beggs and Mercer, 2009; Camiletti et al., 2013; Croft et al., 2017; Kocher and Grozinger, 2011; Oi et al., 2015). By contrast, the bumblebee queen-characteristic compound pentacosane only inhibited the 
reproduction of the workers and not of the queen. It also reduced the aggressive workerworker displays that help to establish the dominance hierarchy in bumblebee colonies (Amsalem et al., 2015). This reaffirms pentacosane as an evolved bumblebee queen pheromone (Holman, 2014; Holman et al., 2017; Van Oystaeyen et al., 2014). The relatively modest effect sizes here imply though that pentacosane may not be the only bioactive, nonvolatile queen pheromone in $B$. terrestris, and that the physical presence of the queen may also be required to induce a full effect (Alaux et al., 2004; Holman et al., 2017; LopezVaamonde et al., 2007). It is noteworthy that QMP did not elicit a reduction in aggressive displays, hence confirming that the behavioral and reproductive regulation of dominance in bumblebee workers are two distinct, yet interacting processes (Amsalem et al., 2015).

The recent finding of cuticular queen pheromones in the honeybee suggests that also the honeybee possesses honest queen signals that are likely derived from ancestral fertility cues (Oliveira et al., 2015; Princen et al., 2019). However, the fact that honeybee-specific QMP shows clear cross-activity in other species like the bumblebee and the fruit fly, and exploits the workers' physiology rekindles the question to what extent the reproduction of social insect workers could be suppressed against their own evolutionary interests, e.g. via the release of substances with hormonal effects (Keller, 2009; Keller and Nonacs, 1993; Kocher and Grozinger, 2011; Oi et al., 2015; Peso et al., 2014; Villalta et al., 2018). At the same time, our data shows that QMP not only inhibited the reproduction of bumblebee workers but also the reproduction of queens, which illustrates a major hurdle for such a scenario. That is, for queen control to be possible, the queens themselves should be immune to the manipulative substances they emit (Holman et al., 2013; Yusuf et al., 2018). In the specific case of honeybee QMP administered to bumblebees, this does not appear to be case. However, queens might well be less sensitive to the sterility-inducing effects of manipulative pheromones than workers, for example due to the queens' much greater fecundity, which could translate in them having a higher threshold at which their reproduction would be inhibited (Holman et al., 2013). Hence, chemical manipulation might - over limited evolutionary time - be possible as part of an evolutionary arms race, at least until workers would evolve resistance mechanisms (Foster et al., 2000; Keller, 2009; Kocher and Grozinger, 2011; Oi et al., 2015; Peso et al., 2014; Yusuf et al., 2018).

An excellent example of such manipulation occurs in the context of intersexual reproductive conflict in the fruit fly (Hollis et al., 2019; Keller, 2009). Here, males transfer over 
100 seminal toxins during mating, which decrease the ability for the female to mate with other males. They do so, however, at a cost of the females' life expectancy, which led them to evolve resistance mechanisms (Arnqvist and Rowe, 2005; Hollis et al., 2019). Previously, one of the compounds of the honeybee QMP, homovanillyl alcohol (HVA), was suggested to act on a preexisting neuromodulatory system in worker bees by binding directly on dopamine receptors as opposed to acting via the sensory system (Beggs et al., 2007; Vergoz et al., 2007). In fruit flies though, mainly the hydroxy-acids, and not HVA, induced the most pronounced antiovarian response (Galang et al., 2019; Princen et al., 2019). Furthermore, possible evidence for arms races is found in anarchistic and socially parasitic honeybees, where reproductive workers mimic the glandular secretions of the queens (Katzav-Gozansky et al., 2003; Martin et al., 2004; Moritz et al., 2002; Niu et al., 2016; Okosun et al., 2017; Okosun et al., 2019; Sole et al., 2002), thereby escaping egg policing and controlling other workers while they themselves appear insensitive to the compounds (Yusuf et al., 2018). Generally though, research suggests that in honeybees worker reproduction is largely made unprofitable by the presence of worker policing, which results in the selective elimination of most worker-laid eggs (Oi et al., 2015; Wenseleers et al., 2004a; Wenseleers and Ratnieks, 2006a). Hence, although some honeybee queen pheromones, and especially QMP, may have evolved via a route of sensory exploitation, the workers are not actually manipulated to remain sterile against their own evolutionary interests.

Overall, our study gives significant new insight into the likely route by which the pheromonal signals that regulate insect eusociality originated in populations, and documents how preadaptations on the part of either the sender or receiver are key to allow such signals to evolve in a step-wise fashion. This confirms a key prediction of animal signaling theory (Bradbury and Vehrencamp, 2011; Stökl and Steiger, 2017; Wyatt, 2014). Our key result - that the non-native honeybee QMP also inhibits egg-laying in both worker and queen bumblebees - adds to the evidence for a route of sensory exploitation. We argue that such exploitation could allow social insect queens to produce compounds that manipulate the workers to remain sterile, but that a major hurdle would be that the queens themselves also have to be immune to such compounds (Holman et al., 2013; Yusuf et al., 2018). At the same time, other queen pheromones, such as hydrocarbon queen pheromones, are most likely derived from ancestral fertility cues and thus have probably evolved via a sender-precursor route (Oi et al., 2015; Van Oystaeyen et al., 2014). Altogether, these data illustrate the diverse paths that 
evolution has taken to produce extremely complex adaptations, including the advanced sociality and the intricate communication systems observed in insect societies.

\section{REFERENCES}

Alaux C, Jaisson P, Hefetz A, 2004. Queen influence on worker reproduction in bumblebees (Bombus terrestris) colonies. Insectes Soc. 51:287-293. doi: 10.1007/s00040-004-0741-5.

Amsalem E, Grozinger CM, Padilla M, Hefetz A, 2015. Chapter 2 - The physiological and genomic bases of bumble bee social behaviour. Adv Insect Physiol. 48:37-93. doi: 10.1016/bs.aiip.2015.01.001.

Arnqvist G, Rowe L, 2005. Sexual conflict: Princeton University Press.

Bates D, Maechler M, Bolker B, Walker S, 2014. Ime4: Linear mixed-effects models using Eigen and S4. R package version. 1:1-23.

Beggs KT, Glendining KA, Marechal NM, Vergoz V, Nakamura I, Slessor KN, Mercer AR, 2007. Queen pheromone modulates brain dopamine function in worker honey bees. Proc Natl Acad Sci USA. 104:2460-2464. doi: 10.1073/pnas.0608224104.

Beggs KT, Mercer AR, 2009. Dopamine receptor activation by honey bee queen pheromone. Curr Biol. 19:1206-1209. doi: 10.1016/j.cub.2009.05.051.

Blomquist GJ, 2010. Biosynthesis of cuticular hydrocarbons. In: Blomquist GJ, Bagnères AG, editors. Insect hydrocarbons: biology, biochemistry, and chemical ecology: Cambridge University Press. p. 35-52.

Blomquist GJ, Bagnères AG, editors. 2010. Insect hydrocarbons: biology, biochemistry, and chemical ecology: Cambridge University Press.

Bradbury JW, Vehrencamp SL, 2011. Principles of animal communication: Sinauer.

Camiletti AL, Awde DN, Thompson GJ, 2014. How flies respond to honey bee pheromone: the role of the foraging gene on reproductive response to queen mandibular pheromone. Naturwissenschaften. 101:25-31. doi: 10.1007/s00114013-1125-3.

Camiletti AL, Percival-Smith A, Croft JR, Thompson GJ, 2016. A novel screen for genes associated with pheromone-induced sterility. Sci Rep. 6:36041. doi: 10.1038/srep36041.

Camiletti AL, Percival-Smith A, Thompson GJ, 2013. Honey bee queen mandibular pheromone inhibits ovary development and fecundity in a fruit fly. Entomol Exp Appl. 147:262-268. doi: 10.1111/eea.12071.

Chapuisat M, 2014. Smells like queen since the Cretaceous. Science. 343-:254-255. doi: 10.1126/science.1249285.

Cho H-C, Abe S, 2013. Is two-tailed testing for directional research hypotheses tests legitimate? J Bus Res. 66:1261-1266. doi: 10.1016/j.jbusres.2012.02.023.

Crewe $\mathrm{R}$, Velthuis $\mathrm{H}, 1980$. False queens: a consequence of mandibular gland signals in worker honeybees. Naturwissenschaften. 67:467-469. doi: 10.1007/BF00405650.

Croft JR, Liu T, Camiletti AL, Simon AF, Thompson GJ, 2017. Sexual response of male Drosophila to honey bee queen mandibular pheromone: implications for genetic studies of social insects. J Comp Physiol A. 203:143-149. doi: 10.1007/s00359-017-1147-y.

Duchateau MJ, Velthuis HHW, 1989. Ovarian development and egg laying in workers of Bombus terrestris. Entomol Exp Appl. 51:199-213. doi: 10.1111/j.1570-7458.1989.tb01231.x.

Foster KR, Ratnieks FL, Raybould AF, 2000. Do hornets have zombie workers? Molecular Ecology. 9:735-742. doi: 10.1046/j.1365-294x.2000.00920.x.

Galang KC, Croft JR, Thompson GJ, Percival-Smith A, 2019. Analysis of the Drosophila melanogaster anti-ovarian response to honey bee queen mandibular pheromone. Insect Mol Biol. 28:99-111. doi: 10.1111/imb.12531.

Hammond RL, Keller L, 2004. Conflict over male parentage in social insects. PLoS Biol. 2:e248. doi: 10.1371/journal.pbio.0020248.

Hollis B, Koppik M, Wensing KU, Ruhmann H, Genzoni E, Erkosar B, Kawecki TJ, Fricke C, Keller L, 2019. Sexual conflict drives male manipulation of female postmating responses in Drosophila melanogaster. Proc Natl Acad Sci U S A. 116:8437-8444. doi: 10.1073/pnas.1821386116.

Holman L, 2014. Bumblebee size polymorphism and worker response to queen pheromone. PeerJ. 2:e604. doi: 10.7717/peerj.604.

Holman L, 2018. Queen pheromones and reproductive division of labor: a meta-analysis. Behav Ecol. 29:1199-1209. doi: 10.1093/beheco/ary023.

Holman L, Leroy C, Jørgensen C, Nielsen J, d'Ettorre P, 2013. Are queen ants inhibited by their own pheromone? Regulation of productivity via negative feedback. Behav Ecol. 24:380-385. doi: 10.1093/beheco/ars174.

Holman L, van Zweden JS, Oliveira RC, van Oystaeyen A, Wenseleers T, 2017. Conserved queen pheromones in bumblebees: a reply to Amsalem et al. PeerJ. 5:e3332. doi: 10.7717/peerj.3332.

Hoover SE, Keeling Cl, Winston ML, Slessor KN, 2003. The effect of queen pheromones on worker honey bee ovary development. Naturwissenschaften. 90:477-480. doi: 10.1007/s00114-003-0462-z.

Jeffreys H, 1961. Theory of probability, 3d ed. Oxford: Clarendon Press.

Kass RE, Raftery AE, 1995. Bayes factors. J Am Stat Assoc. 90:773-795. doi: 10.1080/01621459.1995.10476572. 
Katzav-Gozansky T, Soroker V, Francke W, Hefetz A, 2003. Honeybee egg-laying workers mimic a queen signal. Insectes Soc. 50:20-23. doi: 10.1007/s000400300003.

Keller L, 2009. Adaptation and the genetics of social behaviour. Philos Trans R Soc Lond B Biol Sci. 364:3209-3216. doi: 10.1098/rstb.2009.0108.

Keller L, Nonacs P, 1993. The role of queen pheromones in social insects: queen control or queen signal? Anim Behav. 45:787-794. doi: 10.1006/anbe.1993.1092.

Kocher SD, Grozinger CM, 2011. Cooperation, conflict, and the evolution of queen pheromones. J Chem Ecol. 37:12631275. doi: 10.1007/s10886-011-0036-z.

Le Conte Y, Hefetz A, 2008. Primer pheromones in social hymenoptera. Annu Rev Entomol. 53:523-542. doi: 10.1146/annurev.ento.52.110405.091434.

Lopez-Vaamonde C, Brown RM, Lucas ER, Pereboom JJM, Jordan WC, Bourke AFG, 2007. Effect of the queen on worker reproduction and new queen production in the bumble bee Bombus terrestris. Apidologie. 38:171-180. doi: 10.1051/apido:2006070.

Marsman M, Wagenmakers E-J, 2017. Three insights from a Bayesian interpretation of the one-sided $P$ value. Educ Psychol Meas. 77:529-539. doi: 10.1177/0013164416669201.

Martin SJ, Chaline N, Oldroyd BP, Jones GR, Ratnieks FLW, 2004. Egg marking pheromones of anarchistic worker honeybees (Apis mellifera). Behav Ecol. 15:839-844. doi: 10.1093/beheco/arh089.

Moritz R, Crewe R, 2018. The dark side of the hive: the evolution of the imperfect honeybee: Oxford University Press.

Moritz R, Crewe R, Hepburn H, 2002. Queen avoidance and mandibular gland secretion of honeybee workers (Apis mellifera L.). Insectes Soc. 49:86-91. doi: 10.1007/s00040-002-8284-0.

Murphy R, 2018. On the use of one-sided statistical tests in biomedical research. Clin Exp Pharmacol Physiol. 45:109-114. doi: $10.1111 / 1440-1681.12754$.

Niu D-F, Pirk CW, Zheng H-Q, Ping S, Shi J-H, Cao L-F, Hu F-L, 2016. Reproductive traits and mandibular gland pheromone of anarchistic honey bee workers Apis mellifera occurring in China. Apidologie. 47:515-526. doi: 10.1007/s13592015-0396-4.

Oi CA, Oliveira RC, van Zweden JS, Mateus S, Millar JG, Nascimento FS, Wenseleers T, 2019. Do Primitively Eusocial Wasps Use Queen Pheromones to Regulate Reproduction? A Case Study of the Paper Wasp Polistes satan. Front Ecol Evol. 7:199. doi: 10.3389/fevo.2019.00199.

Oi CA, van Zweden JS, Oliveira RC, Van Oystaeyen A, Nascimento FS, Wenseleers T, 2015. The origin and evolution of social insect queen pheromones: novel hypotheses and outstanding problems. Bioessays. 37:808-821. doi: 10.1002/bies.201400180.

Okosun OO, Pirk CWW, Crewe RM, Yusuf AA, 2017. Glandular sources of pheromones used to control host workers (Apis mellifera scutellata) by socially parasitic workers of Apis mellifera capensis. J Insect Physiol. 102:42-49. doi: 10.1016/j.jinsphys.2017.09.001.

Okosun OO, Yusuf AA, Crewe RM, Pirk CW, 2019. Tergal gland components of reproductively dominant honey bee workers have both primer and releaser effects on subordinate workers. Apidologie. 50:173-182. doi: 10.1007/s13592-018 0628-5.

Oliveira RC, Oi CA, do Nascimento MMC, Vollet-Neto A, Alves DA, Campos MC, Nascimento F, Wenseleers T, 2015. The origin and evolution of queen and fertility signals in Corbiculate bees. BMC Evol Biol. 15:254. doi: 10.1186/s12862-015-0509-8.

Peso M, Elgar MA, Barron AB, 2014. Pheromonal control: reconciling physiological mechanism with signalling theory. Biol Rev. 90:542-559. doi: 10.1111/brv.12123.

Plettner E, Otis GW, Wimalaratne PDC, Winston ML, Slessor KN, Pankiw T, Punchihewa PWK, 1997. Species- and castedetermined mandibular gland signals in honeybees (Apis). J Chem Ecol. 23:363-377. doi: 10.1023/B:JOEC.0000006365.20996.a2.

Princen SA, Oliveira RC, Ernst UR, Millar JG, van Zweden JS, Wenseleers T, 2019. Honeybees possess a structurally diverse and functionally redundant set of queen pheromones. Proc R Soc Lond B Biol Sci.20190517. doi: 10.1098/rspb.2019.0517.

Princen SA, Van Oystaeyen A, Petit C, van Zweden JS, Wenseleers T, 2019. Data from: Cross-activity of honeybee queen mandibular pheromone in bumblebees provides evidence for sensory exploitation. Behavioral Ecology. https://doi.org/10.5061/dryad.c59zw3r3h

Ratnieks FLW, Wenseleers T, 2008. Altruism in insect societies and beyond: voluntary or enforced? Trends Ecol Evol. 23:4552. doi: 10.1016/j.tree.2007.09.013.

Ruxton GD, Neuhäuser M, 2010. When should we use one-tailed hypothesis testing? Meth Ecol Evol. 1:114-117. doi: 10.1111/j.2041-210X.2010.00014.x.

Sibbald ED, Plowright CMS, 2014. Social interactions and their connection to aggression and ovarian development in orphaned worker bumblebees (Bombus impatiens). Behavioural Processes. 103:150-155. doi: 10.1016/j.beproc.2013.11.012.

Sibbald ED, Plowright CMS, 2015. Reproductive potential and its behavioural consequences in orphaned bumblebee workers (Bombus impatiens). Apidologie. 46:618-627. doi: 10.1007/s13592-015-0351-4.

Slessor KN, Kaminski L-A, King GGS, Borden JH, Winston ML, 1988. Semiochemical basis of the retinue response to queen honey bees. Nature. 332:354-356. doi: 10.1038/332354a0.

Slessor KN, Winston ML, Le Conte Y, 2005. Pheromone communication in the honeybee (Apis mellifera L.). J Chem Ecol. 31:2731-2745. doi: 10.1007/s10886-005-7623-9.

Sole CL, Kryger P, Hefetz A, Katzav-Gozansky T, Crewe RM, 2002. Mimicry of queen Dufour's gland secretions by workers of Apis mellifera scutellata and A. m. capensis. Naturwissenschaften. 89:561-564. doi: 10.1007/s00114-002-0370-7. 
Stökl J, Steiger S, 2017. Evolutionary origin of insect pheromones. Curr Opin Insect Sci. 24:36-42. doi: 10.1016/j.cois.2017.09.004.

Van Oystaeyen A, Oliveira RC, Holman L, van Zweden JS, Romero C, Oi CA, d'Ettorre P, Khalesi M, Billen J, Wäckers F, Millar JG, Wenseleers T, 2014. Conserved class of queen pheromones stops social insect workers from reproducing. Science. 343:287-290. doi: 10.1126/science.1244899.

Vergoz V, Schreurs HA, Mercer AR, 2007. Queen pheromone blocks aversive learning in young worker bees. Science. 317:384-386. doi: 10.1126/science.1142448.

Villalta I, Abril S, Cerda X, Boulay R, 2018. Queen control or queen signal in ants: what remains of the controversy 25 years after Keller and Nonacs' seminal paper? J Chem Ecol. 44:805-817. doi: 10.1007/s10886-018-0974-9.

Wenseleers T, Hart AG, Ratnieks FLW, 2004a. When resistance is useless: policing and the evolution of reproductive acquiescence in insect societies. Am Nat. 164:E154-E167. doi: 10.1086/425223.

Wenseleers T, Helanterä H, Alves DA, Dueñez-Guzmán E, Pamilo P, 2013. Towards greater realism in inclusive fitness models: the case of worker reproduction in insect societies. Biol Lett. 9. doi: 10.1098/rsbl.2013.0334.

Wenseleers T, Helanterä H, Hart A, Ratnieks FLW, 2004b. Worker reproduction and policing in insect societies: an ESS analysis. J Evol Biol. 17:1035-1047. doi: 10.1111/j.1420-9101.2004.00751.x.

Wenseleers T, Ratnieks FL, 2006a. Enforced altruism in insect societies. Nature. 444:50. doi: 10.1038/444050a.

Wenseleers T, Ratnieks FLW, 2006b. Comparative analysis of worker reproduction and policing in eusocial Hymenoptera supports relatedness theory. Am Nat. 168:E163-E179. doi: 10.1086/508619.

Wyatt TD, 2014. Pheromones and animal behavior: Chemical signals and signature mixes: Cambridge University Press.

Yusuf AA, Crewe RM, Pirk CWW, 2018. Turning workers into false queens: the role of exogenous pheromones in regulating reproduction in worker honey bees. J Exp Biol. 221:jeb175505. doi: 10.1242/jeb.175505. 


\section{FIGURES}

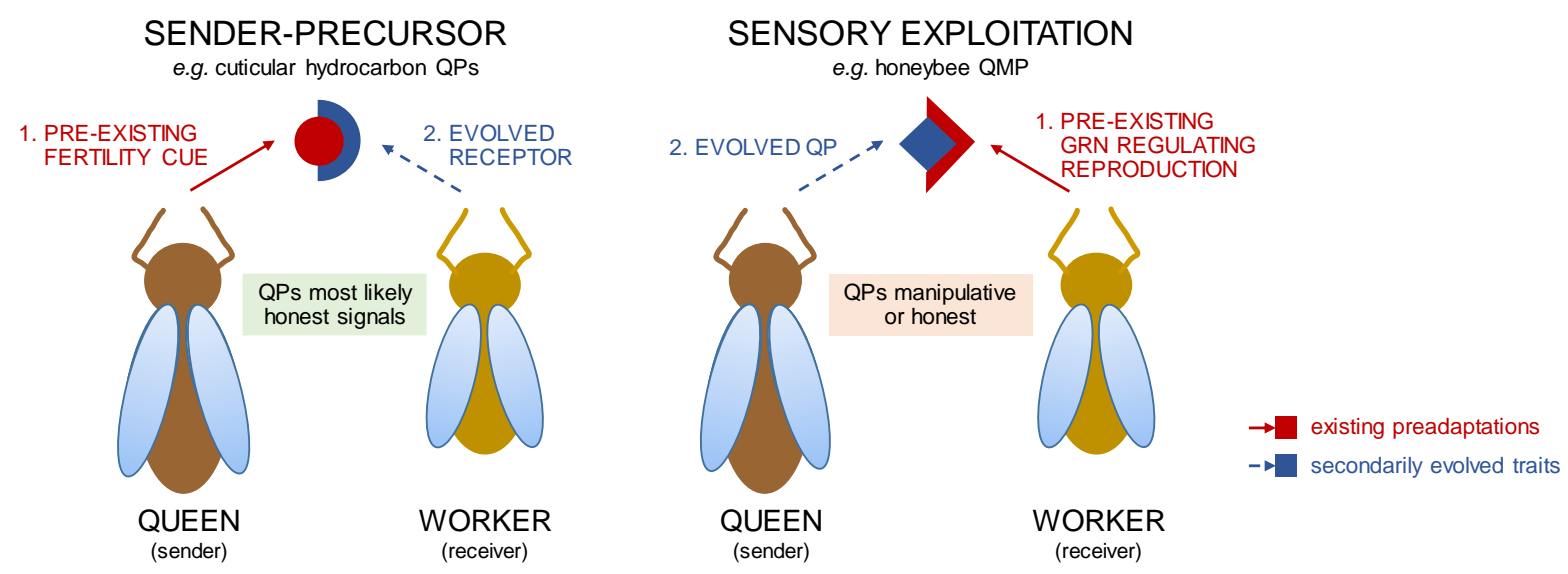

Figure 1. Two alternative evolutionary scenarios by which queen pheromone signals could have evolved and their likely mode-of-action. Two theories currently exist to explain the origin of a chemical signal: the sender-precursor hypothesis (left) and the sensory exploitation hypothesis (right) (Stökl and Steiger, 2017). The former posits that a chemical signal first evolves from a preadaptation on the part the sender, who already produces a precursor of the compound either as a functionless by-product of physiology or to serve another, non-communicative purpose. For the evolution of a full signal, the receiver needs to evolve a way to detect and optimally respond to the provided cue. In the case of queen pheromones (QPs), this scenario is well supported for cuticular hydrocarbon QPs, which generally appear to be derived from ancestral fertility cues (Oi et al., 2015; Oliveira et al., 2015; Van Oystaeyen et al., 2014). Under this scenario, QPs will intrinsically be honest signals of the queen's true fecundity and the workers are expected to respond to such signals to the extent that it serves their evolutionary interests. By contrast, the sensory exploitation hypothesis predicts that QPs could also evolve de novo if they act on a pre-existing gene-regulatory networks (GRNs) that are linked with the regulation of reproduction in the receiving workers. In this case the sender could potentially exploit the existing GRNs to deceive or manipulate the receivers, i.e. the queen would be capable - at least transiently - of manipulating the workers' reproduction against their own evolutionary interests. 

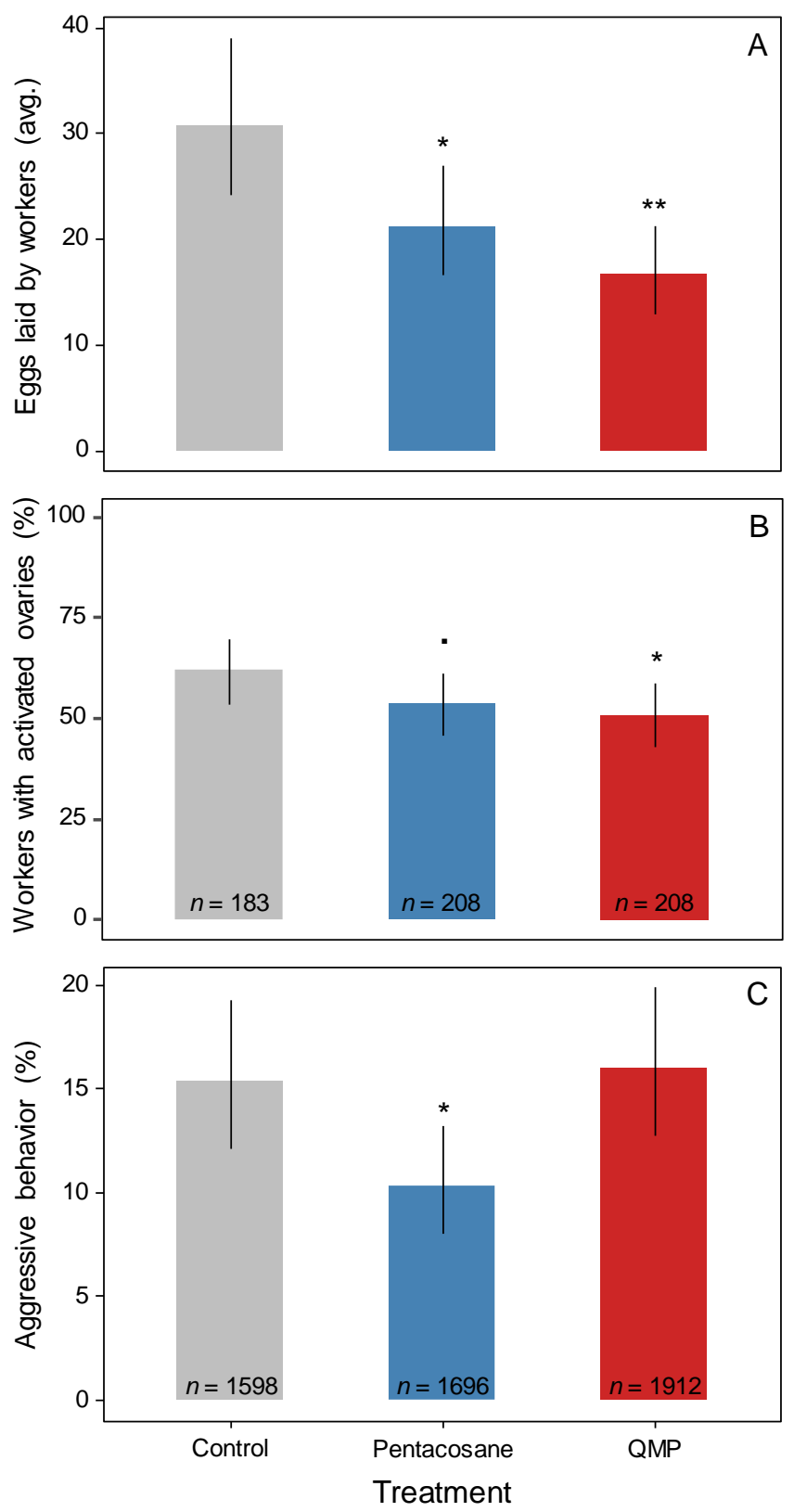

Figure 2. In Bombus terrestris, honeybee QMP inhibits worker egg-laying (A, ratio $=1.86, z$-ratio $=2.96, p=0.003$, Table $\mathrm{S} 1 \mathrm{~b})$ and ovary activation $(\mathrm{B}$, odds ratio $=1.58, z$-ratio $=2.03, p=0.043$, Table $\mathrm{S} 2 \mathrm{~b}$ ) to a similar extent as the native queen pheromone pentacosane $(\mathbf{A}$, ratio $=1.45, z$-ratio $=1.78, p=0.038$, Table $\mathrm{S} 1 \mathrm{~b}$; $\mathbf{B}$, odds ratio $=$ 1.42 , z-ratio $=1.55, p=0.061$, Table S2b) when compared to a solvent-only control. However, only the native $B$. terrestris queen pheromone pentacosane causes a significant reduction in aggressive conflict over reproduction in queenless bumblebee colonies $(\mathbf{C}$, odds ratio $=1.57, z$-ratio $=2.34, p=0.019$, Table S3b; percentage of all behavior observed that was aggressive) compared to the control, whereas no such effect was seen for the nonnative honeybee QMP (odds ratio $=0.95, z$-ratio $=-0.26, p=0.60$ ). Significant differences with the control treatment are indicated by asterisks based on post hoc one-sided FDR corrected $p$ values of the respective Poisson (A) and binomial (B and C) GLMM fits (details in Tables S1a to S3b), marginal significance is denoted by a period and the whiskers denote $95 \%$ confidence bounds. $n=$ total number of eggs laid (A), total number of dissected workers (B) and total number of behavioral interactions recorded (C) per treatment 

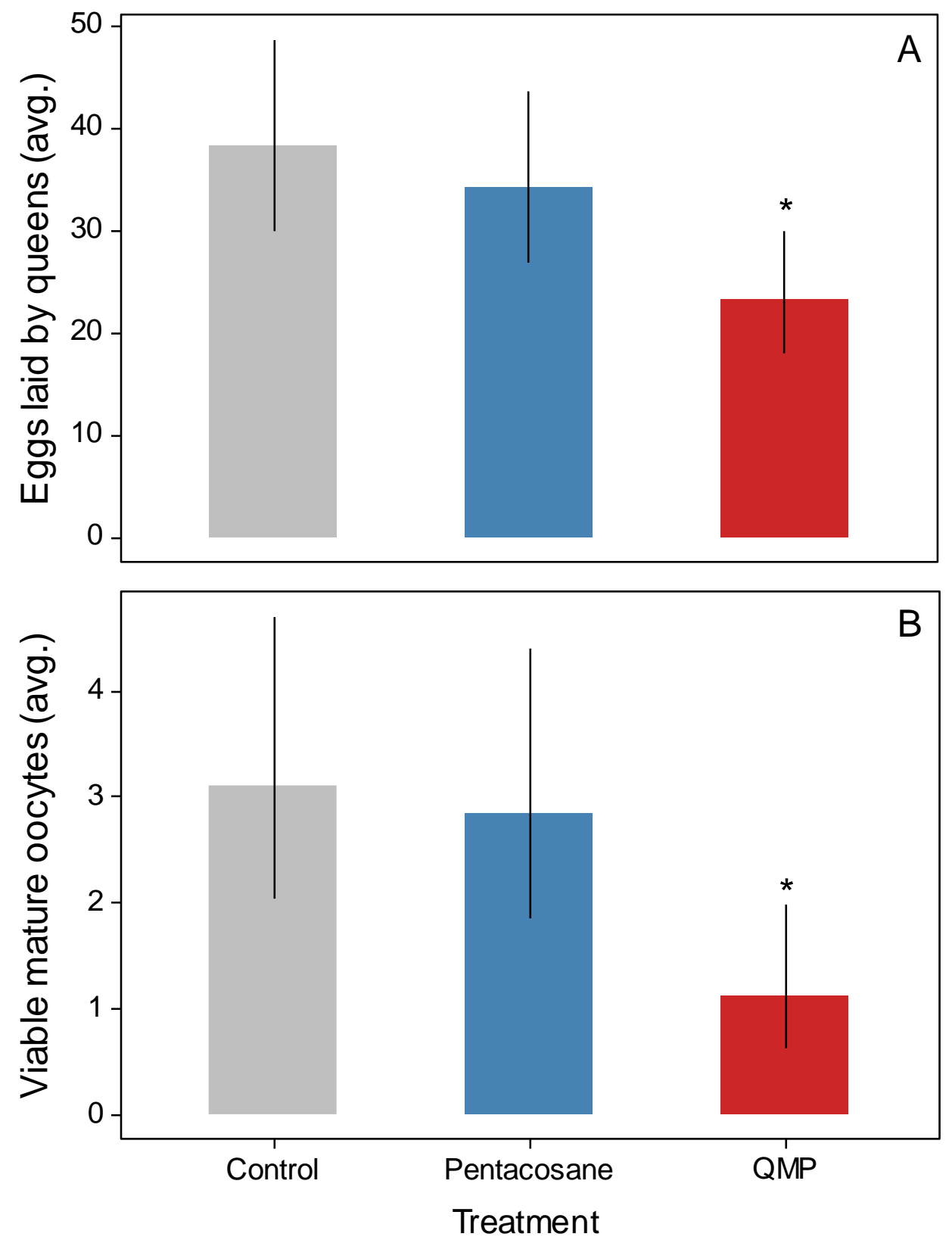

Figure 3. In B. terrestris, honeybee QMP significantly reduces the amount of eggs laid by queens $(A$, ratio $=1.64$, $z$-ratio $=2.34, p=0.019$, Table S4b) and the number of viable mature oocytes in the queens' ovaries (B, ratio = 2.78, z-ratio $=2.47, p=0.014$, Table S5b) compared to the solvent-only control, whereas the native pheromone pentacosane elicits no such effects (A: $z$-ratio $=0.52, p=0.30$, Table S4b; $\mathbf{B}$ : $z$-ratio $=0.24, p=0.41$, Table S5b). Significant differences with the control treatment are indicated by asterisks based on post hoc one-sided FDR corrected $p$ values from two Poisson GLMMs (details in Tables S4a-S5b), and the whiskers denote $95 \%$ confidence bounds. $n=$ total number of eggs laid $(\mathbf{A})$ and total number of viable mature oocytes observed across all dissected queens (B) per treatment 


\section{FUNDING}

This work was supported by the Research Foundation Flanders (grant numbers G.0A51.15, G.0F83.19N) and a Postdoctoral Grant of the Research Foundation Flanders to J.S.v.Z. (grant number $12 \mathrm{Q} 7615 \mathrm{~N})$.

\section{ACKNOWLEDGEMENTS}

We thank An Vandoren for her technical assistance. We would also like to thank the handling editor and two anonymous reviewers for their valuable input.

\section{DATA ACCESSIBILITY}

Analyses reported in this article can be reproduced using the data provided by Princen et al. (2019). 


\section{SUPPLEMENTARY TABLES}

Table S1a. The number of eggs laid by workers was compared across treatments using a Poisson log-link generalized linear mixed model (GLMM) with total number of workers present in the nest at the end of the experiment and worker mortality included as continuous covariates and colony and nest coded as random intercepts. Coefficients, standard errors, $z$-values, one-sided $p$-values and standard deviations of the random effect intercepts are shown. All continuous covariates were standardized using scale() to be able to consistently allow the comparison of their effect. The model was fitted using R's $g / m e r$ function in the Ime4 package using treatment coding whereby the control was coded as the reference level and assuming an unstructured variance-covariance matrix.

\begin{tabular}{rcccc}
\hline Term & Coefficient & SE & z-value & p-value \\
\hline (Intercept: control) & 3.43 & 0.14 & 23.64 & \\
Treatment: pentacosane & -0.37 & 0.21 & -1.78 & 0.038 \\
Treatment: QMP & -0.62 & 0.21 & -2.96 & 0.002 \\
scale(Nr of workers in nest) & -0.03 & 0.088 & -0.37 & 0.35 \\
scale(Mortality) & -0.04 & 0.087 & 0.47 & 0.32 \\
SD(Intercept).Nest & 0.41 & & & \\
SD(Intercept).Colony & $2 \mathrm{e}-5$ & & & \\
\hline
\end{tabular}

Table S1b. FDR-corrected post hoc tests performed on the Poisson log-link GLMM of Table S1a comparing the number of eggs laid by workers in the control condition versus in the pentacosane and QMP treated groups. The ratios of the number of eggs laid in the control versus in each of the two treatments are shown together with the lower and upper $95 \%$ confidence bounds, $z$-ratios and one-sided FDR corrected $p$-values, which give the significance of the mean ratios being greater than 1 and the treatments thus inhibiting worker egg-laying.

\begin{tabular}{rrrrrrr}
\hline Contrast with & Ratio & Lower 95\% CB & Upper 95\% CB & z-ratio & FDR p-value \\
\hline Pentacosane & 1.45 & 1.03 & 2.47 & 1.78 & 0.038 & $*$ \\
QMP & 1.86 & 1.32 & 3.15 & 2.96 & 0.003 & $* *$ \\
\hline
\end{tabular}


Table S2a. The proportion of workers with activated ovaries was compared across treatments using a binomial logit-link generalized linear mixed model (GLMM) with body size, total number of workers present in the nest at the end of the experiment and worker mortality included as continuous covariates and colony and nest coded as random intercepts. Coefficients, standard errors, $z$-values, one-sided $p$-values and standard deviations of the random effect intercepts are shown. All continuous covariates were standardized using scale() to be able to consistently allow the comparison of their effect. The model was fitted using R's glmer function in the Ime4 package using treatment coding whereby the control was coded as the reference level and assuming an unstructured variance-covariance matrix.

\begin{tabular}{|c|c|c|c|c|c|}
\hline Term & Coefficient & $S E$ & z-value & p-value & \\
\hline (Intercept: control) & 4.95 & 0.21 & 2.37 & & \\
\hline Treatment: pentacosane & -0.35 & 0.23 & -1.55 & 0.061 & . \\
\hline Treatment: QMP & -0.46 & 0.23 & -2.03 & 0.021 & $*$ \\
\hline scale(Body size) & 0.29 & 0.097 & 2.94 & 0.002 & $* *$ \\
\hline scale(Nr of workers in nest) & -0.61 & 0.12 & -5.29 & $6 e-8$ & $* * *$ \\
\hline scale(Mortality) & -0.29 & 0.11 & -2.61 & 0.005 & $* *$ \\
\hline SD(Intercept).Nest & $4 e-5$ & & & & \\
\hline SD(Intercept).Colony & 0.38 & & & & \\
\hline
\end{tabular}

Table S2b. FDR-corrected post hoc tests performed on the binomial logit-link GLMM of Table S2a comparing the proportion of workers with activated ovaries in the control versus in the pentacosane and QMP treatment groups. The odds ratios of the workers having activated ovaries in the control condition versus in the two treatment groups are show together with the lower and upper $95 \%$ confidence bounds, $z$-ratios and one-sided FDR corrected $p$-values, which give the significance of the mean odds ratios being greater than 1 and the treatments thus inhibiting worker ovary activation.

\begin{tabular}{rrrrrrr}
\hline Contrast with & Odds ratio & Lower 95\% CB & Upper 95\% CB & z-ratio & FDR p-value \\
\hline Pentacosane & 1.42 & 0.98 & 2.54 & 1.55 & 0.061 & $\cdot$ \\
QMP & 1.58 & 1.10 & 2.86 & 2.03 & $0.043 *$ \\
\hline
\end{tabular}


Table S3a. The proportion of recorded behavioral actions that were aggressive was compared across treatments using a binomial logit-link generalized linear mixed model (GLMM) with total number of workers and worker mortality included as continuous covariates and colony and nest coded as random intercepts. Coefficients, standard errors, $z$-values, one-sided $p$ values and standard deviations of the random effect intercepts are shown. All continuous covariates were standardized using scale() to be able to consistently allow the comparison of their effect. The model was fitted using R's glmer function in the Ime4 package using treatment coding whereby the control was coded as the reference level and assuming an unstructured variance-covariance matrix.

\begin{tabular}{rcccc}
\hline Term & Coefficient & SE & z-value & p-value \\
\hline (Intercept: control) & -1.71 & 0.17 & -10.23 & \\
Treatment: pentacosane & -0.45 & 0.19 & -2.34 & 0.0096 \\
Treatment: QMP & 0.047 & 0.18 & 0.26 & 0.60 \\
scale(Total nr of workers) & -0.18 & 0.10 & -1.88 & 0.030 \\
scale(Mortality) & -0.033 & 0.09 & 0.37 & 0.36 \\
SD(Intercept).Nest & 0.34 & & & \\
SD(Intercept).Colony & 0.32 & & & \\
\hline
\end{tabular}

Table S3b. FDR-corrected post hoc tests performed on the binomial logit-link GLMM of Table S3a comparing the level of aggression in the control condition versus in the pentacosane and QMP treated groups. The odds ratios of displayed behaviour being aggressive in the control condition versus in the two treatment groups are shown together with the lower and upper $95 \%$ confidence bounds, $z$-ratios, and one-sided FDR corrected $p$-values, which give the significance of the mean odds ratios being greater than 1 and aggression being reduced by the queen pheromone treatments.

\begin{tabular}{rrrrrrr}
\hline Contrast with & Odds ratio & Lower 95\% CB & Upper 95\% CB & z-ratio & FDR p-value \\
\hline Pentacosane & 1.57 & 1.14 & 2.54 & 2.34 & $0.019 *$ \\
QMP & 0.95 & 0.70 & 1.49 & -0.26 & 0.60 \\
\hline
\end{tabular}


Table S4a. The number of eggs laid by queens was compared across treatments using a Poisson log-link generalized linear mixed model (GLMM) with queen ID coded as random intercept. Coefficients, standard errors, $z$-values, one-sided $p$-values and standard deviations of the random effect intercepts are shown. The model was fitted using R's glmer function in the Ime 4 package using treatment coding whereby the control was coded as the reference level and assuming an unstructured variance-covariance matrix.

\begin{tabular}{|c|c|c|c|c|c|}
\hline Term & Coefficient & $S E$ & z-value & p-value & \\
\hline (Intercept: control) & 3.64 & 0.15 & 24.75 & & \\
\hline Treatment: pentacosane & -0.11 & 0.21 & -0.52 & 0.30 & \\
\hline Treatment: QMP & -0.50 & 0.21 & -2.34 & 0.010 & $* *$ \\
\hline SD(Intercept).Queen ID & 0.43 & & & & \\
\hline
\end{tabular}

Table S4b. FDR-corrected post hoc tests performed on the Poisson log-link GLMM of Table S1a comparing the number of eggs laid by queens in the control condition versus in the pentacosane and QMP treated groups. The ratios of the number of eggs laid in the control versus in each of the two treatments are shown together with the lower and upper $95 \%$ confidence bounds, $z$-ratios and one-sided FDR corrected $p$-values, which give the significance of the mean ratios being greater than 1 and the treatments thus inhibiting queen egg-laying.

\begin{tabular}{rrrrrr}
\hline Contrast with & Ratio & Lower 95\% CB & Upper 95\% CB & z-ratio & FDR p-value \\
\hline Pentacosane & 1.11 & 0.79 & 1.89 & 0.52 & 0.30 \\
QMP & 1.64 & 1.16 & 2.81 & 2.34 & $0.019 *$ \\
\hline
\end{tabular}


Table S5a. The number of visible viable oocytes in the queens' ovaries was compared across treatments using a Poisson loglink generalized linear mixed model (GLMM) with queen ID coded as random intercept. Coefficients, standard error, $z$-values, one-sided $p$-values and standard deviations of the random effect intercepts are shown. The model was fitted using R's $g / m e r$ function in the Ime4 package using treatment coding whereby the control was coded as the reference level and assuming an unstructured variance-covariance matrix.

\begin{tabular}{|c|c|c|c|c|c|}
\hline Term & Coefficient & $S E$ & z-value & p-value & \\
\hline (Intercept: control) & 1.13 & 0.25 & 4.47 & & \\
\hline Treatment: pentacosane & -0.08 & 0.35 & -0.24 & 0.41 & \\
\hline Treatment: QMP & -1.02 & 0.41 & -2.47 & 0.007 & $* *$ \\
\hline SD(Intercept).Queen ID & 0.54 & & & & \\
\hline
\end{tabular}

Table S5b. FDR-corrected post hoc tests performed on the Poisson log-link GLMM of Table S1a comparing the number of visible viable oocytes in the queens' ovaries in the control condition versus in the pentacosane and QMP treated groups. The ratios of the number of eggs laid in the control versus in each of the two treatments are shown together with the lower and upper $95 \%$ confidence bounds, $z$-ratios and one-sided $F D R$ corrected $p$-values, which give the significance of the mean ratios being greater than 1 and the treatments thus reducing the number of viable oocytes in the queens' ovaries.

\begin{tabular}{rrrrrr}
\hline Contrast with & Ratio & Lower 95\% CB & Upper 95\% CB & z-ratio & FDR p-value \\
\hline Pentacosane & 1.09 & 0.64 & 3.47 & 0.24 & 0.41 \\
QMP & 2.78 & 1.53 & 14.74 & 2.47 & $0.014 *$ \\
\hline
\end{tabular}


Table S6a. The number of dead workers in each nest at the end of the experiment was compared across treatments using a Poisson log-link generalized linear mixed model (GLMM) with total number of workers (living + dead) added as a continuous covariate and colony and nest coded as random intercepts. Coefficients, standard errors, $z$-values, one-sided $p$-values and standard deviations of the random effect intercepts are shown. The model was fitted using R's glmer function in the Ime 4 package using treatment coding whereby the control was coded as the reference level and assuming an unstructured variance-covariance matrix.

\begin{tabular}{rcccc}
\hline Term & Coefficient & SE & z-value & p-value \\
\hline (Intercept: control) & 0.82 & 0.25 & 3.31 & \\
Treatment: pentacosane & -0.08 & 0.29 & -0.29 & 0.39 \\
Treatment: QMP & -0.02 & 0.29 & -0.06 & 0.48 \\
Scale(Total workers (living + dead)) & 0.25 & 0.14 & 1.76 & 0.04 \\
SD(Intercept).Nest & 0.05 & & & \\
SD(Intercept). Colony & 0.38 & & & \\
\hline
\end{tabular}

Table S6b. FDR-corrected post hoc tests performed on the Poisson log-link GLMM of Table S0a comparing the number of dead workers in the control condition versus in the pentacosane and QMP treated groups. The ratios of the number of dead workers in the control versus in each of the two treatments are shown together with the lower and upper $95 \%$ confidence bounds, $z$-ratios and one-sided FDR corrected $p$-values, which give the significance of the mean ratios being greater than 1 and the treatments thus increasing worker mortality.

\begin{tabular}{rrrrrr}
\hline Contrast with & Ratio & Lower 95\% CB & Upper 95\% CB & z-ratio & FDR p-value \\
\hline Pentacosane & 0.92 & 0.69 & 2.54 & -0.29 & 0.48 \\
QMP & 0.98 & 0.65 & 2.38 & -0.06 & 0.48 \\
\hline
\end{tabular}




\section{SUPPLEMENTARY FIGURES}

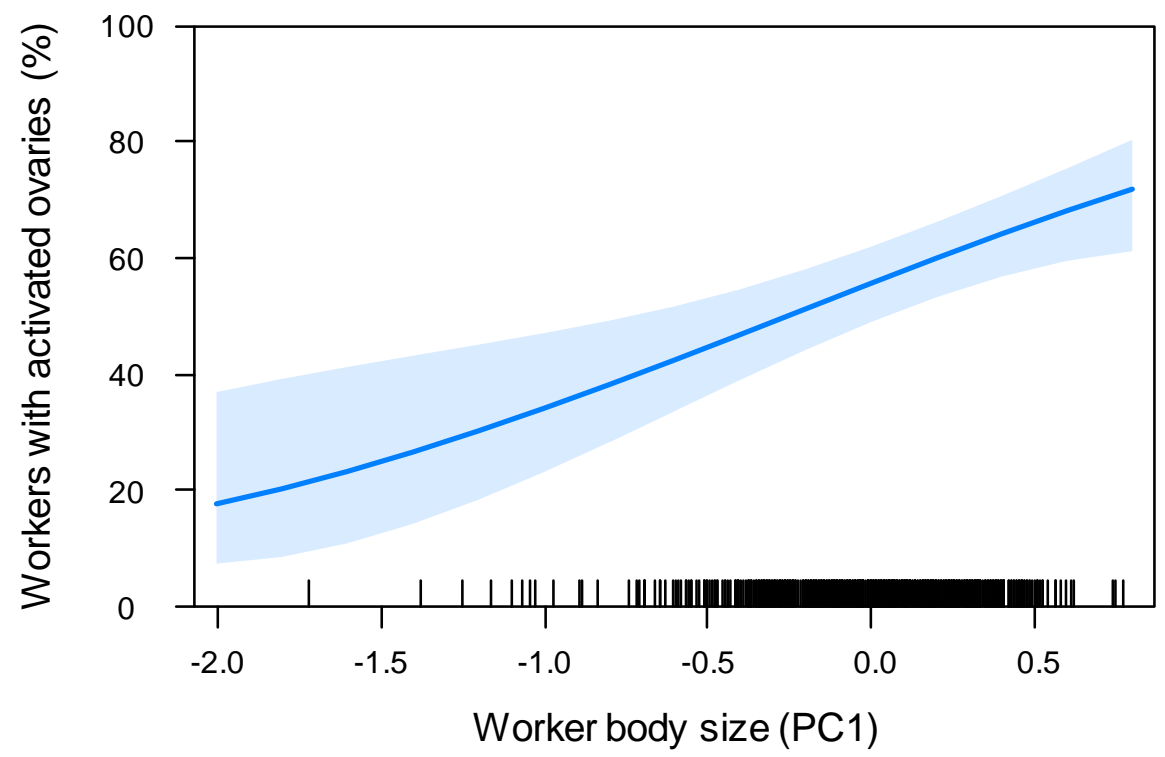

Figure S1. Effect plot depicting the average partial effect of worker body size on the proportion of workers with activated ovaries ( $z=0.29, p=0.002$; Table S2a). Bigger workers had a higher probability of having activated ovaries across treatments. The worker body size measure used was calculated from the first PCA axis of an analysis of head width and mean length of the frontal wings' marginal cells (PCA projection used: Body size $=0.748 *$ (head width $(\mathrm{mm})$ - mean head width) $+0.663 *$ (marginal cells length $(\mathrm{mm})$ - mean marginal cell length) $=0.748 *$ (head width $(\mathrm{mm})-4.160)+0.663 *$ (marginal cell length $(\mathrm{mm})-2.955)$, see supplemental $\mathrm{R}$ script). The light blue band indicates the $95 \%$ confidence bounds. The black ticks on the $x-$ axis represent the marginal distribution of the independent covariate, i.e. a rug plot.

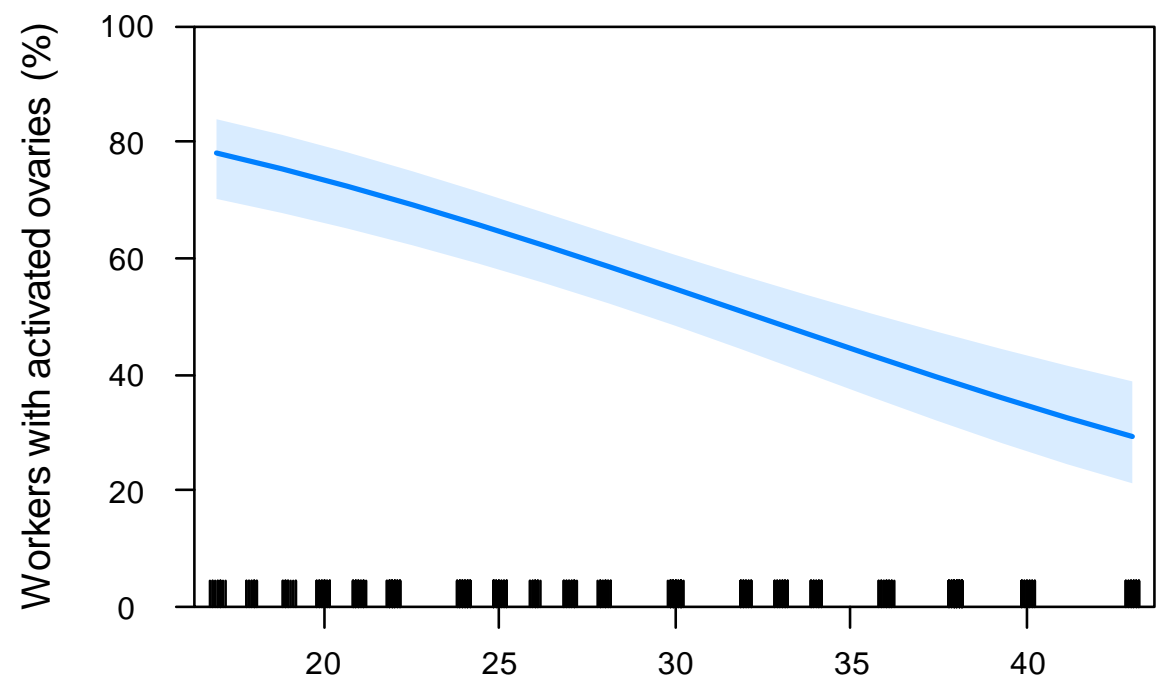

Number of workers in the nest

Figure S2. Effect plot depicting the average partial effect of the number of workers present in the nest after seven days on the proportion of workers with activated ovaries $(z=-0.61, p=6 \mathrm{e}-08$; Table S2a). When more workers were present in the nest at the end of the experiment, the percentage of workers with activated ovaries went down. This suggests that colonies where greater number of new workers eclosed had smaller proportions of egg-laying workers because the newly eclosed workers were most likely pre-reproductive. Alternatively, it could be that each worker group had a similar total number of workers with activated ovaries, irrespective of the total number of workers in the nest. The light blue band indicates the $95 \%$ confidence bounds. The black ticks on the $x$-axis represent the marginal distribution of the independent covariate, i.e. a rug plot. 


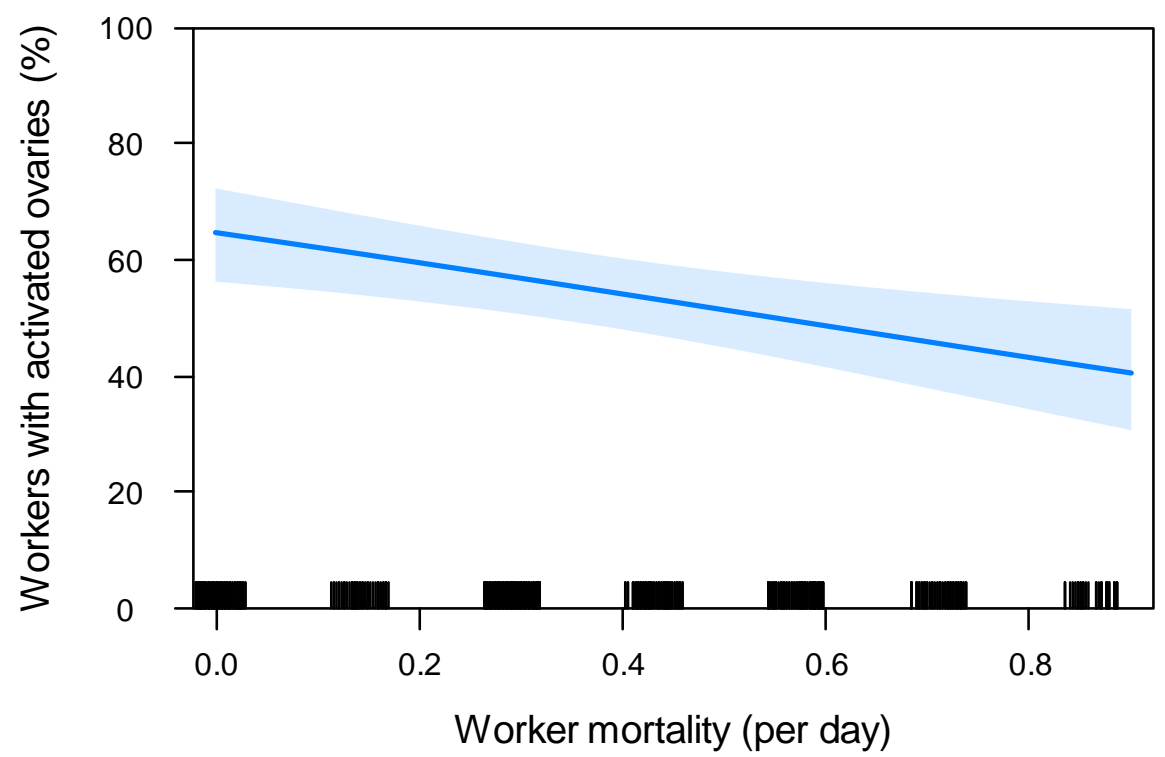

Figure S3. Effect plot depicting the average partial effect of the worker mortality (average number of workers that died per day) on the proportion of workers with activated ovaries $(z=-0.29, p=0.005$; Table S2a). Nests that experienced higher worker mortality contained fewer workers with activated ovaries, perhaps due to increased stress. The light blue band indicates the $95 \%$ confidence bounds. The black ticks on the $x$-axis represent the marginal distribution of the independent covariate, i.e. a rug plot.

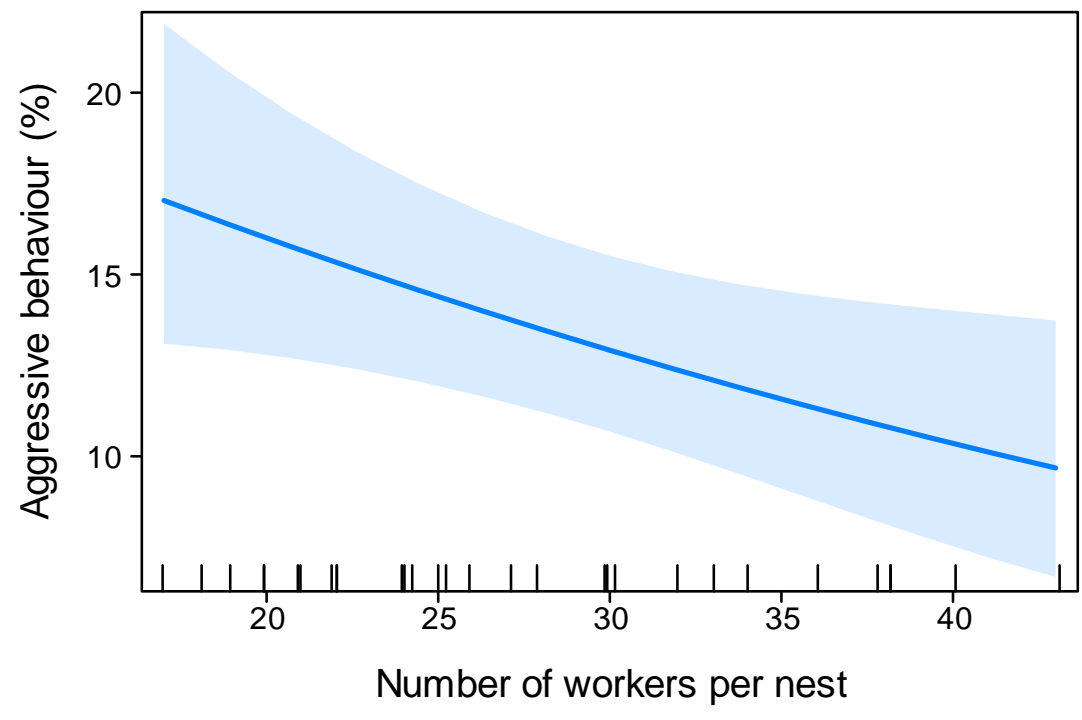

Figure S4. Effect plot depicting the average partial effect of the number of workers present in the nest after seven days on the proportion of observed aggressive behavioral interactions ( $z=-0.18, p=0.030$; Table S3a). Aggression decreased when there were more workers present in the nest at the end of the experiment. This seems logical since Fig S1b shows that the average number of reproductive workers was approximately constant across nests with a different total number of workers. Hence, the aggression linked with reproductive competition would also be expected to quantitatively stay the same, whereas the number of other non-aggressive behaviours would increase in function of nest size. The light blue band indicates the $95 \%$ confidence bounds. The black ticks on the $\mathrm{x}$-axis represent the marginal distribution of the independent covariate, i.e. a rug plot. 